\title{
Object and Gesture Recognition to Assist Children with Autism during the Discrimination Training
}

\author{
Eduardo Quintana ${ }^{1}$, Catalina Ibarra ${ }^{2}$, Lizbeth Escobedo ${ }^{2}$, \\ Monica Tentori ${ }^{1}$, and Jesus Favela ${ }^{1}$ \\ ${ }^{1}$ Department of Computer Science, CICESE, México \\ \{equintan, mtentori, favela\}@cicese.mx \\ ${ }^{2}$ School of Computer Science, UABC, México \\ \{cibarra, lescobedo\} @uabc.edu.mx
}

\begin{abstract}
Teachers prompt children with autism to redirect their attention to the object discrimination training and reduce the time they spend "off task". In this paper, we describe MOBIS, a mobile augmented reality application enabling multi-modal interaction to provide guidance to students with autism during the object discrimination training. The system uses a vision-based object recognition algorithm to associate visual and verbal prompts to the object being discriminated (i.e., "object of interest"). The results of a performance evaluation of the system show that the object recognition component achieves an accuracy of $90 \%$, processing an image every 0.5 seconds. Accelerometers placed on objects of interest are used to detect interaction gestures with an accuracy of $87 \%$. The performance of both algorithms is sufficient to support the object discrimination training in real-time.
\end{abstract}

Keywords: Augmented reality, object recognition, multimodal interaction.

\section{Introduction}

Teachers of children with autism ${ }^{1}$ use real objects and paper-based visual supports during the object discrimination training (Fig 1 right). These supports, along with prompts from the teacher help students match a real object to its corresponding visual support. Paper-based visual supports, however, are not interactive and appealing to students that spend considerable time "off task". Technological interventions that provide interactive visual supports (e.g., vSked [6]) help teachers to keep students "on task" [6]. However, these tools still lack some realism to help students generalize from the classroom to other environments. Thus, a new type of interactive visual supports capable of augmenting the physical form of a traditional object with digital information is needed.

\footnotetext{
${ }^{1}$ For simplicity of reading we will refer as students to the children with autism attending to the object discrimination training at Pasitos - a specialized clinic attending to close to 60 lowfunctioning students http: / /www. pasitos . org
} 
One possible solution to this problem is the use of Augmented Reality (AR) due its capability to seamlessly superimpose digital information on real objects. Previous work has demonstrated that AR technology [3, 10] is suitable for children with autism. Additionally, AR enables the automated record-keeping, relieving teachers of this onerous burden. In this paper, we present the design and implementation of MOBIS, a mobile augmented reality system that combines the benefits of digital and physical supports, and helps teachers to keep students "on task". MOBIS relies on a vision-based object recognition component using the Speeded-Up Robust Features (SURF) algorithm [1], and uses an algorithm for recognizing interactions gestures to facilitate the record-keeping. Teachers can upload new images to the system at any time and use them to annotate new objects of interest. In the reminder of the paper we motivate the problem, describe the system and present the results of its evaluation.

\section{Related Work: Ubicomp in Support of Autism}

The Ubicomp community has proposed several applications to support the training of students with autism. Over the last decade, ubiquitous technologies that use visual supports have mainly supported children with autism to manage their visual schedules [5] or remediate their speech and language disabilities [2], serving most of the time as an augmentative communication tool [2]. For example, the GreceApp or DynaVox [2] uses a speech-generating system running in tablets, that enables children with autism to communicate effectively by building semantic sequences from relevant images to form sentences based on the Picture Exchange Communication System (PECS). Other ubiquitous solutions use virtual characters as communication intermediaries between children with autism and their peers [9].

Several projects have researched the use of interactive displays to support the use of visual schedules inside the classroom (e.g., vSked [6]), and mobile technology for the generalization of skills (e.g., MOSOCO [3, 10]). The deployment studies of these applications have proven that ubiquitous solutions in support of students with autism reduces the quantity of educator-initiated prompts [3] and enables skills generalization in real life situations [3]. This work shows that there is great potential in the use of ubiquitous tools in support of the training of students with autism. However, leaves open questions as to how the benefits students gained using digital visual supports could be obtained when using real objects -often used during the discrimination training.

\section{Discrimination Training for Autism}

To supplement our understanding from our literature review we conducted a qualitative study at Pasitos. For a period of $\mathbf{3}$ months we conducted 13 semistructured interviews with 11 teachers working at this clinic and we complemented our interviews with $\mathbf{7 5}$ hours of passive observation.

The results of our study uncover current practices around the object discrimination training. Teachers at Pasitos use the combined blocking procedure [11] to teach a 
student how to discriminate objects. This method demands from the student to conduct repetitions about a particular task, called trials. The task for each trial involves the discrimination of one object or one color. Most of the observed teachers conduct a total of 10 trials per object per student, and after 10 completed trials the teacher will change the object or color used to discriminate. A trial is considered complete if the student successfully chooses the object being discriminated without the need of teacher-initiated prompts. Teacher-initiated prompts include:

- verbal, a spoken instruction or follow-up to a request,

- physical or model, hand-over-hand physical interaction or demonstrate the behavior being requested, and/or

- visual, sometimes called positional, placement of objects in such a way that the correct choice is closer to the student or pointing to the correct choice.

Each trial is reinforced with a reward (e.g., tickles, spoken congratulations). To exemplify how teachers conduct an object discrimination lesson at Pasitos here we present a scenario.

Alexa, a low-functioning child with autism, is learning how to discriminate the orange fruit. The teacher Adie starts the trial placing in the table four paper-based laminated cards each one with different fruits (Fig 1). Then, Adie starts the first trial of 10 and asks Alexa to grab the card that contains an orange. Alexa grabs the chips card instead and starts playing with it. So Adie prompts her saying, "Alexa! Grab the orange!", and points towards the orange card. Alexa loses concentration and starts looking around. Adie grabs Alexa's hand to help her grab the orange card saying: "Alexa! Grab the orange card!" Alexa grabs the orange card and gives it to Adie. Adie rewards her by saying: "Good job!" while marking the trial.

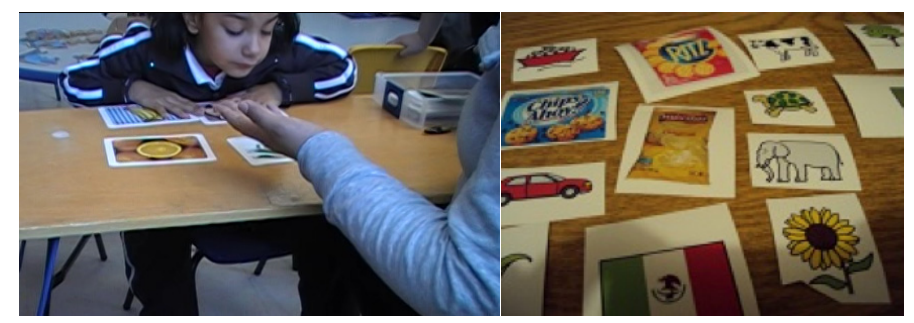

Fig. 1. A student with autism attending to an object discrimination lesson (left) Paper-based visual supports used during the object discrimination training (right)

This scenario shows the workload teachers have during the training, and how much effort they invest in keeping the student "on task" constantly giving prompts while maintaining a detailed record of students' interactions with objects.

\section{The Mobile OBject Identification System (MOBIS)}

We used the results of the study to iteratively design and implement our system MOBIS, a mobile augmented reality application that combines the benefits of visual 
and physical supports to help teachers cope with the challenges faced during the object discrimination training. MOBIS consists of three interfaces: (1) one running in a tablet teachers use to set up the therapy and monitor each trial, (2) a second one running in a smartphone a student uses as a "visor"[4] to uncover visual and verbal prompts added on top of physical objects (Fig 2 right), and (3) the third one is a Tangible User Interface (TUI) housing accelerometers that could be attached to the objects being discriminated to detect students' interaction gestures to facilitate the record-keeping for the teacher. MOBIS architecture includes 4 main subsystems: the Tag Manager, the coordinationNotepad, an extended version of the Ambient Notification System (ANS) [8], and Augmented Objects using accelerometers to detect students' interaction gestures [7]. The Tag Manager and the ANS rely on an object recognition module that uses SURF algorithm [1], robust against variations in illumination, viewpoint, rotation and scale.

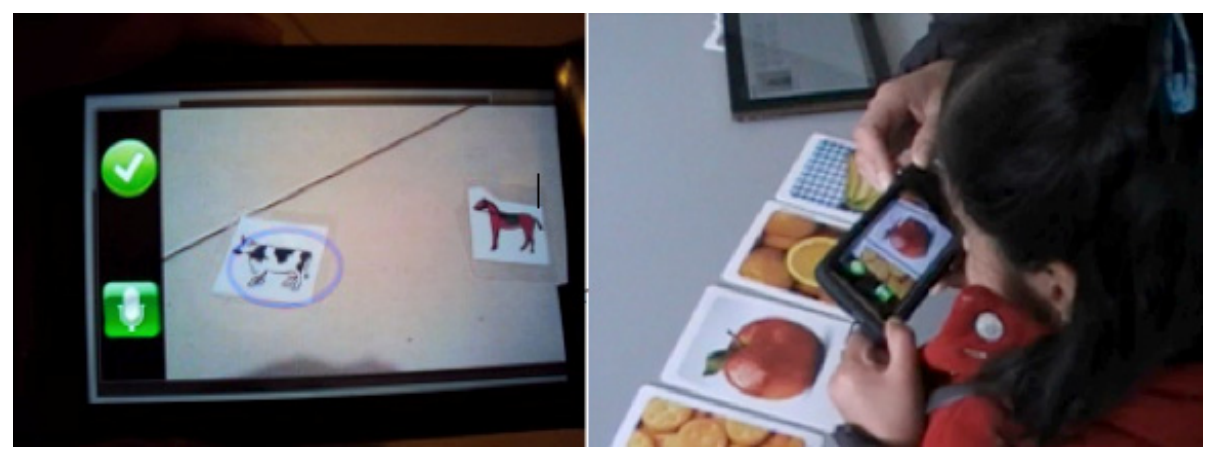

Fig. 2. MOBIS giving visual prompts to a student with autism

\subsection{The Tag Manager}

Teachers use the Tag Manager to upload captured photographs of relevant objects, and associate tags to photos and tagged photos to therapies. To create a new tag, teachers select the object and associate a visual support (e.g., a circle) to an audio or a text message. This message will be displayed to the student with autism as a prompt superimposed over the detected object (Fig 2 left). This subsystem is deployed on a server and is also responsible for feature extraction and object recognition.

The Tag Manager displays all the photographs associated to that therapy from which the teacher can select one that shows the object to which s/he wants to associate the tag. The system automatically displays other photographs where the same object is recognized, allowing the teacher to select the object and increase the number of features that will be used to compare with the query image. To retrieve these images, first, the SURF algorithm selects the interest points (IPs) of the "object of interest" (i.e., the object being discriminated) at distinctive locations in the image (e.g., corners, blobs). The most valuable property of an IP detector is its repeatability, i.e. whether it reliably finds the same IPs under different viewing conditions. Then, the IP-neighborhood is represented with a 64-feature vector distinctive and robust to 
noise, detection errors, and geometric and photometric deformations. Finally, the Euclidean distance is used to match feature vectors from all images.

\section{2 coordinationNotepad}

Teachers use the coordinationNotepad on a tablet, to select the object the student will need to discriminate, the number of trials, and the amount of prompts and rewards available per trial. Teachers first select the number of students that will participate in a trial, as the system supports multiple users. Then the teacher selects the object the student will learn to discriminate. The ANS Tag Search Engine (see 4.3.2), to improve performance, only considers the tagged objects that match the object selected by the teacher. Next, the teacher selects the number of prompts, including visual and audio prompts, vibration, and a combination of the three, to be provided to students. The level of prompting will depend on the functioning level of the student and should be faded out as the student executes the skill being taught without needing object-initiated prompts. Having these different forms of visualization support multiple modes of interaction. Then, the teacher selects the rewards associated to each trial and to the complete activity. Finally, the teacher selects the number of trials per activity, and initiates the activity. This will activate the ANS Client running in the student's smartphone. The coordinationNotepad is paired up with the objects augmented with accelerometers to enable the assisted-automatic capture of students' interactions with the "objects of interest".

\subsection{The Ambient Notification System}

The Ambient Notification System (ANS) runs on the smartphone used by the students. It continuously captures images using the camera in the mobile device and sends them to the server. When the server notifies the mobile application that a tag has been found, the ANS alerts the student with an audio notification and/or vibrating. Then the ANS superimposes the tag on the image of the detected physical object. Students uncover the tag using the camera of the smartphone as a visor to physical objects (Fig 2 right). This application mimics educator-initiated prompts supporting multiple modes of interaction based on teachers' configuration and available tagged images. First, the system shows the student his/her photograph on the screen's top left and a traffic light on its right indicating they have to wait for a teacher's request (Fig 2 left). When the teacher activates the trial, the system exchanges the traffic light with the image of the object the teacher is asking the student to discriminate. To achieve this functionality ANS executes two main processes:

- Request tags: This process runs continuously in the background and it is only suspended to allow the server to process the client's request. The client application sends the captured photograph to the server, which performs a matching process against the associated tagged object the teacher is using to discriminate. The client has a simplified copy of the tag repository (keeping only audio and text files, and leaving out IPs files), which is used to prompt the 
students according to pre-configured modes of interaction (audio, visual prompt, vibration, etc.).

- Update tag repository: This process is executed every minute. The client sends an "update request" message to the server, which checks a changelog file to monitor changes in the Tag Manager.

When a new photograph is received from the smartphone, the server extracts the interest points and matches them to the IPs of the "object of interest" selected by the teacher for each therapy.

\subsection{Augmented Objects}

Objects augmented with accelerometers [7] attached to the "objects of interest" assist teachers with the capture of students' interactions with objects detecting when the student grabs, releases and shakes an object. If the teacher does not receive a correct response from the object, the system will start prompting the student based on the preconfigured modes of interaction. When the teacher receives the correct student's response the system displays the reward to the student. Augmented objects work in pair with the coordinationNotePad, assisting the teacher to monitor the therapy and allowing her to manually correct the detected interaction gesture if needed.

The approach for gesture recognition uses windows of $0.5 \mathrm{sec}$ containing 25 accelerometer readings. The algorithm uses the mean, variance and RMS of these readings as features and a linear classifier, to detect three interaction gestures: grabbing, shaking and releasing. To evaluate the performance of this approach we conducted an experiment with 8 participants using the augmented objects imitating the interaction gestures used during a therapy for a period of 3 minutes. Each interaction gesture was continuously performed for at least $30 \mathrm{sec}$. The results of this evaluation shows our approach is able to detect the grabbing gesture with $82.5 \%$ accuracy, releasing with $85.70 \%$ and shaking with $92.95 \%$, for an average of $87.08 \%$.

\section{Evaluation}

In this section we present the main results from the performance evaluation of the object recognition component of MOBIS used to define appropriate operation parameters for the system and to evaluate the system under realistic conditions of use.

We used a digital camera to collect a dataset of 197 800x600 images portraying the use of different objects. Photographs were taken from different angles in the classroom. From this set, 39 images were used as queries and 158 were used to create 32 tags associated to objects considered relevant to one particular training activity. Additional 800x480 images were captured by the children using a smartphone when conducting a training session and are used to evaluate the performance under real operating conditions.

We used the 39 query images in the first dataset to assess the accuracy of the system. In 35 of the 39 queries the system retrieved the correct tag, resulting in an accuracy of $89.7 \%$. In this test the search is performed in all 32 tags. However, during 
the therapy the student is asked to discriminate only one object at a time and the search can be performed only with this tag.

We compared the system performance when searching for only one to five tags (formed by all IPs obtained from all the objects selected from those images where the object appears), with all the tags created for a given therapy (32), and with all 159 images available in the dataset. On average, with 1 to 5 tags with 39 queries, the server takes 1.7 seconds to process each query, 5.4 seconds with 32 tags and 11 seconds searching in all images. Clearly, adapting the search in real-time to look only for the tag of interest for the task at hand considerably improves performance.

We conducted additional tests with 6 sequences of images gathered in real-time when students used MOBIS during an object discrimination lesson (Figure 4). As it can be seen, several images are blurry or partially covered. This happens because students continuously move the smartphone and often block the camera with a finger while holding the smartphone. In the sequence shown in Figure 4 the student was asked to grab the card with the red train, which was accurately recognized in the first two images. We estimated the accuracy of the system with 6 different sequences with a total of 205 images, in which the student was asked to grab 6 different objects. In contrast with the evaluation described above, in this case the comparison was made only between the photographs used as query and a single tag associated to the object of interest. The average accuracy obtained was $71.6 \%$. The ground truth was defined as any image recognized by a person as the target object. For instance, of the 6 images in Figure 4, a human can recognize the object in 4 of them. False negatives however, were often followed by true positives in the next or subsequent query where the object might be more visible. Of the 205 images processed, only once was an object not recognized by the system while being somewhat visible in 5 consecutive images, and 3 times it was not detected in 3 consecutive images. The queries were performed on average every 0.7 seconds. Thus, the object was correctly recognized within 2 seconds, except in one case, which required $3.5 \mathrm{~s}$. The performance of the system improved with respect to the previous test $(0.7 \mathrm{~s}$ vs. $1.7 \mathrm{~s})$ since the images obtained from the smartphone have a lower resolution, the query is compared with only 1 tag (instead of 1 to 5), and also because less IPs are detected when images are blurry. Using the 205 images as queries the system produced 7 false positives, but none of them were consecutive. Thus, restricting the notification of a new object found to two consecutive true positives would eliminate false notifications. This makes sense during the actual therapy, since a new image is processed in less than one second and we don't want the system to notify the student of the presence of an object when the camera is moved randomly from one place to another, but rather when it is pointed to an object decisively.

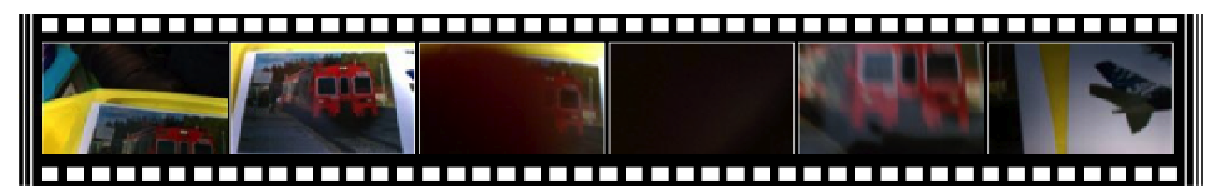

Fig. 3. Sequence of images obtained from the ANS Client during a discrimination therapy 


\section{Conclusion}

We presented MOBIS, a multimodal system assisting students during the object discrimination training. MOBIS combines the benefits of digital and visual supports using augmented reality technology relying on a vision-based object recognition algorithm and augmented objects for gesture recognition. Several experiments were conducted to establish the appropriate parameters of MOBIS by adequately balancing performance and accuracy. We used contextual information (i.e., the activity and location) to reduce the search space. Our results show that MOBIS achieves an adequate performance to efficiently support the object discrimination training using a smartphone.

We are currently running a deployment study of MOBIS in three classrooms at Pasitos to measure the efficacy of the system and its impact in current practices.

\section{References}

1. Bay, H., Ess, A., Tuytelaars, T., Van-Gool, L.: Speeded-up robust features. Computer Vision and Image Understanding 110(3), 346-359 (2008)

2. Binger, C.: Classroom-Based Language Goals and Intervention for Children Who Use AAC: Back to Basics. Perspective on Augmentative and Alternative Communication 17, 20-26 (2008)

3. Escobedo, L., Nguyen, D., Hayes, G., Boyd, L., Rangel, A., García, D., Hirano, S., Tentori, M.: MOSOCO: A Mobile Assistive Tool to Support Children with Autism Practicing Social Skills in Real-Life Situations. In: CHI 2012. ACM, Austin (2012)

4. García-Macías, J.A., Alvarez-Lozano, J., Estrada, P.E., Aviles-Lopez, E.: Browsing the Internet of Things with Sentient Visors. IEEE Computer 44(5), 46-52 (2011)

5. Hayes, G.R., Hirano, S., Marcu, G., Monibi, M., Nguyen, D.H., Yeganyan, M.: Interactive Visual Supports for Children with Autism. Personal and Ubiquitous Computing 14(7) (2010)

6. Hirano, S.H., Yeganyan, M.T., Marcu, G., Nguyen, D.H., Boyd, L., Hayes, G.R.: vSked: evaluation of a system to support classroom activities for children with autism. In: 28th CHI 2010. ACM Press, Atlanta (2010)

7. Ibarra, C., Escobedo, L., Tentori, M.: Smart objects to support the discrimination training of children with autism. In: UBICOMP 2012 (submitted, 2012)

8. Quintana, E., Favela, J.: Ambient Notifications as Memory Aids for People Suffering from Dementia. In: 5th Intl. Conf. on Ubiquitous Computing and Ambient Intelligence (UCAMI 2011), Riviera Maya, Mexico (2011)

9. Tartaro, A., Cassell, J.: Playing with virtual peers: bootstrapping contingent discourse in children with autism. In: International Conference of the Learning Sciences (2008)

10. Tentori, M., Hayes, G.R.: Designing for interaction immediacy to Enhance Social Skills of Children with Autism. In: UBICOMP 2010, Denmark, Copehaguen, pp. 51-60 (2010)

11. Williams, G., Pérez-González, L.A., Muller, A.: Using a combined blocking procedure to teach color discrimination to a child with autism. Journal of Applied Behavior Analysis 38(4), 555-558 (2005) 